\title{
Developing and Testing the Acceptability of Therapeutic Food for the Management of Severely Malnourished Children
}

\author{
Michael Wiafe Akenteng ${ }^{1}$, Reginald Adjetey Annan ${ }^{1}$, Herman Erick Lutterodt ${ }^{2}$, Victoria Pearl Dzogbefia ${ }^{1}$ and \\ Charles Apprey ${ }^{1}$ \\ 1. Department of Biochemistry and Biotechnology, Kwame Nkrumah University of Science and Technology, Kumasi 233, Ghana \\ 2. Department of Food Science and Technology, Kwame Nkrumah University of Science and Technology, Kumasi 233, Ghana
}

\begin{abstract}
Therapeutic foods for the treatment of malnutrition in children under age 5 years are mostly imported from developed countries by developing countries like Ghana. This comes with huge cost and puts pressure on health authorities when these supplies are limited. The objective of this study was therefore to develop and evaluate therapeutic food made from locally agricultural produce (banana, coconut water, soybean, sugar and vegetable oil) for the management of severely acute malnutrition in children under age 5 years. The proximate, mineral and microbial analyses were conducted on the developed product to ensure the standard falls within the requirement of WHO Protocol for the treatment of malnutrition in children. Sensory analysis was also done on the product using children under five years as the target group. The chemical analysis of product revealed energy and protein content of $95.96 \mathrm{kcal}$ and $1.61 \mathrm{~g}$ per $100 \mathrm{~g}$ respectively, and percentage concentration of Calcium (0.74), Magnesium (1.09), Potassium (4.79), Sodium (0.17), Phosphorus (0.28), Iron (0.0080), Copper (0.0064), Manganese (0.0089) and Zinc (0.0174). The microbiological examination indicated aerobic plate count $(20 \mathrm{cfu} / \mathrm{mL})$, yeast count $(8.5 \mathrm{cfu} / \mathrm{mL})$, coliform count $(3 \mathrm{cfu} / \mathrm{mL})$ and zero count for both mould and $E$. Coli. This shows that, the developed product has the potential of treating malnutrition effectively in children under five years old.
\end{abstract}

Key words: Therapeutic food, malnutrition.

\section{Introduction}

Globally, malnutrition claims millions of lives and causes disability every year [1]. Worldwide, undernutrition continues to affect several millions of people, particularly living in developing countries [2]. Internationally, undernutrition is observed as a major public health issue [3]. According to UNICEF, a child dies every 15 seconds as a result of malnutrition and infection [4]. The UNICEF statistics shows that the percentage of children between 1-59 months who live in least developed countries are agonized with moderate and severe stunting, wasting and underweight are 38, 10 and 23 respectively [5]. While

Corresponding Author: Michael Wiafe Akenteng, lecturer and registered/licensed dietician, research fields: malnutrition, dietary management of chronic diseases, therapeutic food formulation and product development. in the developed countries undernutrition is declining progressively, the cases of undernutrition continue to rise in third world countries globally [6].

Undernutrition in the wider perspective is persistent adherence to inefficient nutrition in terms of the quantity and quality of the food intake coupled with infections [7]. Undernutrition can either be acute or chronic depending on the duration of the infection and/or inadequate nutrient intake by the child. Children with chronic undernutrition have prolonged episodes of inadequate nutrition and repeated infections leading to stunting. Acute undernourished children have relatively short period of inadequate food intake and infections which leads to wasting and in severe cases result in oedema [8-10].

Children mostly become malnourished due to infections, poor feeding, poor hygiene, poor education, 
inadequate food or nutrient intake. UNICEF has categorised the causes of undernutrition into three: immediate, underlying and basic causes. The immediate causes are the infection and inadequate food intake. The underlying causes are the inappropriate care and feeding practices, household food insecurity, and poor hygiene and inadequate health facilities. The basic causes are unemployment, illiteracy, low income, and inadequate human, economic and social capital, sociocultural and political issues $[4,11]$.

Undernutrition has both short and long term consequences on the child and even to the unborn generation. The expected short term consequences are morbidity which can lead to either disability or mortality. The long term consequences will be the effect on adult: short stature, cognitive impairment, and low economic and reproductive performance, high risk of metabolic and cardiovascular diseases [4, 12].

Therapeutic foods are given to undernourished children either during the stabilization phase or rehabilitation phase. Therapeutic food is any food that is nutritionally fortified to have high energy and nutrients to meet the needs of the severely malnourished child [13]. Some known therapeutic foods are F-75, F-100, F-135, BP 100, plumpy nut, plumpy doz, "Khichri”. Usually, during the rehabilitation phase the malnourished children are given RUTF which is high in energy, protein, electrolytes (Potassium, Magnesium and low in sodium) and contains other vitamins and minerals [14].

There are major issues about treatment cost, acceptability, foreign ingredients importation, delivery time and both economic and physical access to imported therapeutic foods. The objective of this study was therefore to develop and evaluate therapeutic food made from locally agricultural produce (banana, coconut water, soybean, sugar and vegetable oil) for the management of severely acute malnutrition in children under age 5 years.

\section{Materials and Methods}

\subsection{Materials}

The ingredients used for the formulation of the samples were banana, coconut water, soybean, sugar and olive oil.

\subsection{Preparation of Materials}

Banana: The banana was washed and peeled manually. It was then sliced into reasonable sizes and weighed.

Coconut water: The coconut water was obtained by cracking the coconut and the water was poured for consumption. The coconut water was then weighed.

Soybean flour: The soy bean was sorted out and cleaned. It was then roasted for about 20-30 minutes. The roasted soya was milled into flour to obtain smooth and consistent particles.

Soybean flour milk: 1 cup ( $250 \mathrm{~g}$ ) of the flour was mixed with 4-5 cups $(1,000 \mathrm{~mL}-1,250 \mathrm{~mL})$ of water. The milk was boiled on low fire. When it started boiling, it was time to simmer for about 30-40 minutes. The milk was allowed to cool and a sticky film was removed from the top of the milk after cooling.

\section{Product formulation:}

Half of the soya bean milk was mixed with half of the coconut water in a blender.

The banana, sugar and vegetable oil were then added and blended between two (2) to three (3) minutes.

The remaining soya bean milk and coconut water were added and blended again between two (2) to three (3) minutes.

The process was used for all the samples labelled 519, 586 and 588.

\section{Questionnaire design:}

One questionnaire was used to gather data on the three products but had distinct codes to distinguish them among the products. The questionnaire was in a tabular form and respondents were only to tick as they examine the products. The attributes used were 
appearance, consistency, sweetness and flavour/taste and the 9 point hedonic scale was used to assess the like and dislike of product 519, 586 and 588.

Procedure: the researcher explained the purpose of the study to the mothers/caregivers. The mothers and children were made to wash their hands. The consumers were given napkin and tissue papers. The food was served in a $4 \mathrm{oz}$ plastic cup identified by three code number and a disposable spoon. Each sample cup was identified by three digit codes random number. Each respondent evaluated all the three samples using same questionnaire type but with different codes.

\subsection{Serving Order}

The three samples were served to each mother or caregiver monadic order. The first sample served was removed before the second sample and the second sample served was removed before the third sample. Respondents were made to rinse their mouth with clean water before serving each sample. There was a time delay of 5 minutes between servings. Three to four consumers completed the sensory evaluation at every 20 minutes. The serving order was randomized.

\subsection{Subjects}

Fifty lactating mothers or cargivers were recruited from the Reproductive and Child Health at the Manhyia District Hospital in the Kumasi Metropolis. The lactating mothers or caregivers were screened on the following criteria: (1) Mothers who had children between the ages of 6-59 months and (2) the children were either healthy or malnourished with or without complications.

\subsection{Sensory Evaluation}

The mothers or caregivers were asked to sign a consent form. They rated the samples on the following set of attributes namely appearance, consistency, sweetness and flavour/taste using a standard 9-point hedonic scale, in which 1 was equal to dislike extremely and 9 was equal to like very much.

\subsection{Chemical Analysis}

The proximate analysis of the sample was determined by using the standard procedures of Association of Official Analytical Chemist. The oven drying method was used to determine the moisture content [15]. Protein was determined by Kjedahl method [15]. Fat was determined by the Rose-Gottlieb method [16]. Ash was determined by dry ashing method by placing the sample in a muffle furnace and ignited for twelve hours at $550{ }^{\circ} \mathrm{C}$ [17]. Carbohydrate was determined by calculating the percent difference after moisture; protein, fat and ash were measured. Total energy was determined by calculating the water factor. The macro minerals such as potassium and sodium were determined by the flame photometry while magnesium and calcium were determined by EDTA titration method. The micro minerals which included iron, manganese, copper and zinc were determined by the atomic absorption spectroscopy (AAS).

\subsection{Microbiological Analysis}

Sample Preparation (Homogenization and Serial Dilutions): About $10 \mathrm{~g}$ of product 519 was aseptically weighed into $90 \mathrm{~mL}$ of sterile salt peptone solution (XPS) containing $0.1 \%$ peptone and $0.8 \%$ sodium chloride with $\mathrm{pH}$ adjusted to 7.2 and homogenized in the Stomacher (mode 1 4001, Seward Medical) for 30 seconds at normal speed. This provided $10^{-1}$ dilution. This was vortex for about 2 minutes to ensure uniform mixing. Using a sterile pipette, $1 \mathrm{~mL}$ of the $10^{-1}$ dilution was pipette into $9 \mathrm{~mL}$ of sterile salt peptone water to obtain $10^{-2}$ dilution. This procedure was repeated for $10^{-3}, 10^{-4}, 10^{-5}$ and $10^{-6}$ dilutions. Further, from appropriate tenfold serial dilution, $1 \mathrm{~mL}$ aliquot of each dilution $\left(10^{-1}, 10^{-2}, 10^{-3}, 10^{-4}, 10^{-5}\right.$ and $\left.10^{-6}\right)$ was inoculated into sterile Petri dish plates and the appropriate media added for enumeration and isolation. 

Severely Malnourished Children

After appropriate incubation, dilutions with 30-300 colonies were selected and counted. The number of colony-forming units per $\mathrm{mL}$ (cfu mL ${ }^{-1}$ ) of food was calculated by multiplying the number of bacteria by the dilution. All analysis was done in duplicate for reliability of results.

Enumeration of Aerobic Mesophiles (Viable Plate Count): Aerobic mesophiles were counted by the pour plate method using plate count agar medium (OXOID CM 325). The plates were incubated at $30{ }^{\circ} \mathrm{C}$ for 72 hours (NMKL No. 86, 2013).

Enumeration of Yeast and Moulds: Yeasts and moulds were enumerated by the pour plate method on Dichloran Rose Bengal Chloramphinicol (DRBC) medium (Oxoid CM0727; Oxoid Ltd., Basingstoke, Hampshire, UK) to which $1 \%$ chloramphinicol in absolute ethernol was added as supplement to suppress bacteria growth. The $\mathrm{pH}$ was adjusted to 5.6 and incubated at $25{ }^{\circ} \mathrm{C}$ for 3-5 days in accordance with ISO 21527-1 (2008).

Enumeration and Isolation of Total Coliform: Coliform bacteria were counted by the pour plate method using Tryptone Soya Agar medium (OXOID CM131) and adjusted to $\mathrm{pH} 7.3$ and overlaid with Violet Red Bile agar (OXOID CM 107) with $\mathrm{pH}$ adjusted to 7.4 and incubated at $37^{\circ} \mathrm{C}$ for 24 hours. Colonies were confirmed using Brilliant Green Bile broth (OXOID CM 31) at $\mathrm{pH}$ of 7.4 and incubated at $37^{\circ} \mathrm{C}$ for 24 hours (NMKL No. 44, 2004).

Enumeration of Escherichia Coli: E. coli bacteria were counted by the pour plate method using Trypsin Soya agar medium (OXOID CM131) adjusted to the pH 7.3 and overlaid with Violet Red Bile agar (OXOID CM 107) with $\mathrm{pH}$ adjusted to 7.4 and incubated at $44{ }^{\circ} \mathrm{C}$ for 24 hours. Colonies were confirmed using E.C. broth (OXOID CM 853) with $\mathrm{pH}$ adjusted to 6.9. Colonies that produced gas were confirmed for Indole production. This was done by sub-culturing into Tryptone water and incubated at $44{ }^{\circ} \mathrm{C}$ for 24 hours. Indole test was done by putting a drop of Convac reagent into the culture. Red ring colouration at the surface of Tryptone indicated Indole positive (NMLK No. 125, 2005).

\subsection{Statistical Analysis}

The data gathered on the acceptability test using the questionnaire designed for the samples were analysed using SPSS V16 [18]. The samples' differences and ranking were determined using descriptive analysis and ANOVA. Analysis of variance was employed on the samples means of the 9-point hedonic scale on the attribute of concern: appearance, consistency, sweetness and taste. Statistical significance on the attributes were further analyzed to see where mean differences existed using Post Hoc Test-LSD at 95\% confidence interval $(p<0.05)$.

\section{Result and Discussion}

\subsection{Sensory Evaluation}

The recent uses of affective analysis or hedonic test in consumer preferences have been established to be highly efficient tool in product development and production that will sell in substantial quantities or permit higher pricing [19]. P-519 was most preferred in terms of consistency (7), sweetness (7) and flavour/taste (8). But the appearance of P-586 was highly rated among the three samples (Table 1 ). The varied choice of the attributes resulting in the highest and least preferred of the product might be as a result of the diverse cultural backgrounds, experiences, attitudes and habits of respondents [20]. A study showed that different cultures affected the acceptance of flavours and taste of healthy juices [21]. The overall average based on the attributes such as appearance, consistency, sweetness and flavour of P-519, P-586 and P-588 were 29, 27 and 27 respectively (Table 1). This suggests that P-519 had the highest respective averages on the attributes ranked with the 9-Point Hedonic scale. The ANOVA findings revealed that there was no statistical significance among the three products in terms of appearance $(p=0.109)$, sweetness $(p=0.214)$ and 

Severely Malnourished Children

flavour/taste ( $p=0.634$ ) because neither of the $p$-value was less than 0.05 (Table 1). It indicated that the appearance, sweetness and flavour/taste of P-519, P-586 and P-588 were similar. This could be attributed to the fact that they were made from the same ingredients and their respective proportions of the ingredients for the formulation of the each of the product were not much. However, there was statistical significance $(p<0.05)$ among the three products in terms of consistency $(p=0.02)$. This could be attributed to the considerable difference particularly in the quantities of banana and coconut water used.

\subsection{Comparison of the Nutrient Content of P-519 with Other Therapeutic Foods}

The study revealed that potassium, magnesium, calcium, sodium, zinc and copper content of P-519 per
$100 \mathrm{~g}$ met the proposed nutrient composition range for RUTF but the phosphorus and iron content did not. It had higher nutrient content than F-75 and good amount of sodium, zinc, magnesium and copper when compared to F-100 and F-135 respectively which are already being used in severe malnutrition management (Table 2). Even though, P-519 does not contain added combined vitamin and mineral mix it has shown to be a good nutrient competition with these therapeutics food compared. Generally, it indicates that the ingredients that were used in the formulation of P-519 were rich in these macro and micro minerals. In a normal population there is difference in nutrient requirements because of the variation in body weight, body composition and physiological state [23]. Even though the nutrient content of P-519 did not meet all the nutrients recommendations for RUTF, it is possible

Table 1 Average and mean acceptability scores of the Products.

\begin{tabular}{lllll}
\hline Attributes & P-519 (Mean) & P-586 (Mean) & P-588 (Mean) & $P$-value \\
\hline Appearance & $7(7.20)$ & $8(7.70)$ & $7(7.20)$ & 0.109 \\
Consistency & $7(7.14)$ & $6(6.34)$ & $7(6.80)$ & 0.02 \\
Sweetness & $7(6.66)$ & $6(6.20)$ & $6(5.92)$ & 0.214 \\
Flavour/taste & $8(7.58)$ & $7(7.44)$ & $7(7.24)$ & 0.634 \\
Overall & $29(28.58)$ & $27(27.68)$ & $27(27.16)$ & \\
\hline
\end{tabular}

Table 2 Comparison of the nutrient content of P-519 with other therapeutic foods.

\begin{tabular}{lllllll}
\hline $\begin{array}{l}\text { Nutrients } \\
\text { (Concentration) }\end{array}$ & P-519 & F-75 & F-100 & F-135 & RUTF & Plumpy Nut \\
\hline Protein (g/100 g) & $1.61(1.61)$ & 0.9 & 2.9 & 3.3 & $10 \%-12 \%$ & 13.6 \\
Energy (kcal/100 g) & $95.69(95.96)$ & 75 & 100 & 135 & $520-550$ & 545 \\
Carbohydrate (g/100 g) & $15.27(15.27)$ & - & - & - & - & - \\
Fat (g/100 g) & $3.16(3.16)$ & - & - & - & $45 \%-60 \%$ & 35.7 \\
Ash (g/100 g) & $0.46(0.46)$ & - & - & - & - & - \\
Moisture (g/100 g) & $79.5(79.5)$ & - & - & - & $2.5 *$ & - \\
Potassium (mg/100 g) & $4,790(4.79)$ & 4.0 & 6.3 & 7.7 & $1,100-1,400$ & 1,111 \\
Magnesium (mg/100 g) & $1,090(1.09)$ & 0.43 & 0.73 & 0.8 & $80-140$ & 92 \\
Calcium (mg/100 g) & $740(0.74)$ & - & - & - & $300-600$ & 300 \\
Sodium (mg/100 g) & $170(0.17)$ & 0.6 & 1.9 & 2.2 & $290 *$ & $<290$ \\
Phosphorus (mg/100 g) & $280(0.28)$ & - & - & - & $300-600$ & 300 \\
Iron (mg/100 g) & $7.98(0.008)$ & - & - & - & $10-14$ & 11.5 \\
Zinc (mg/100 g) & $17.4(0.0174)$ & 2.0 & 2.3 & 3.0 & $11-14$ & 14 \\
Copper (mg/100 g) & $6.45(0.0065)$ & 0.25 & 0.25 & 0.25 & $1.4-1.8$ & 1.8 \\
Manganese (mg/100 g) & $8.90(0.0089)$ & - & - & - & - & -
\end{tabular}

Source: RUTF figures adapted from WHO (2007) [3]; F-75, F-100 and F-135 figures adapted from WHO (2003) [9]; Plumpy nut figures adapted from Action Contre Le Faim (2011) [22].

*Maximum. 
for some severely malnourished children who will be treated with it to recover since it meets the standards nutritional recommendation of therapeutic foods as prescribed by World Health Organization.

\subsection{Comparing the Nutrient Content of P-519 with Colostrum and Mature Milk}

Comparing the energy, protein and fat among P-519, colostrums and mature milk there are differences. The energy content of P-519 is $95.96 \mathrm{kcal} / 100 \mathrm{~g}$ which is higher than both colostrums and mature milk which has $58 \mathrm{kcal}$ and 58-72 kcal/100 g respectively. Colostrum has protein content of $2.3 \mathrm{~g} / 100 \mathrm{~g}$ higher than that in mature milk and P-519 however P-519 has higher protein than mature milk. The fat content among the three foods are respectively are mature milk (4.2 g/100 g), product 519 (3.16 g/100 g) and colostrums (2.9), which shows that mature milk has higher fat than P-519. P-519 is rich in both macro and micro minerals than colostrums and breast milk (Table 3). Besides the biological and physical factors that could affect the breast milk production, the method of nutrient analysis could have caused the changes in the differences in the nutrients.

\subsection{Comparison of the Nutrient Content of P-519 with} Dietary Reference Intake for Infants and Children

The nutrient content of P-519 relative to the Dietary
Reference Intake for infants and children showed that P-519 has energy and carbohydrate content of 95.96 kcal and 15.27/100 g respectively which means that infants and children would have to eat extra quantities of P-519 to meet their energy and carbohydrate need for the day whereas the protein content of P-519 per $100 \mathrm{~g}$ which is $1.61 \mathrm{~g}$ is adequate for both infants and children for a day. With the exception of the Recommended Dietary Allowance (RDA) of iron for infants (0.5-1 year), the trace minerals content of P-519 per $100 \mathrm{~g}$ far exceeds all the RDAs and Adequate Intakes (AIs) for infants and children. The macro minerals relation of Product 519 to the dietary reference intake for infants and children revealed that, potassium (4,790 mg), magnesium (1,090 $\mathrm{mg}$ ) and calcium (740 mg) content of P-519 per $100 \mathrm{~g}$ are enough to meet the needs of the infants and children per day. Even though P-519 has phosphorus content $280 \mathrm{mg} / 100 \mathrm{~g}$ which meets the dietary reference intake for infants (0-0.5 years; $0.5-1$ years) but it is less than that required by children (1-3 years) per day. The sodium content of P-519 per $100 \mathrm{~g}$ will be sufficient for infants between 0-0.5 years but it is less than enough for infants between 0.5-1 years and children between 1-3 years respectively (Table 4), which indicates that extra amount of P-519 has to be consumed by these infants and children within those age groups to meet their phosphorus and sodium needs for the day.

Table 3 Comparing the nutrient content of P-519 with colostrum and mature Milk.

\begin{tabular}{llll}
\hline Nutrients (per 100 g) & P-519 & Colostrum & Mature milk \\
\hline Energy (Kcal) & 95.96 & 58 & $58-72$ \\
Protein (g) & 1.61 & 2.3 & 0.9 \\
Fat (g) & 3.16 & 2.9 & 4.2 \\
Potassium (mg) & 4,790 & 74 & 58 \\
Magnesium (mg) & 1,090 & 3.4 & 3.0 \\
Sodium (mg) & 170 & 48 & 18 \\
Calcium (mg) & 740 & 23 & 28 \\
Phosphorus (mg) & 280 & 14 & 15 \\
Iron (mg) & 7.98 & $0.045(45 \mu \mathrm{g})$ & $0.04(40 \mu \mathrm{g})$ \\
Zinc (mg) & 17.4 & $0.54(540 \mu \mathrm{g})$ & $0.12(120 \mu \mathrm{g})$ \\
\hline
\end{tabular}

Source: Colostrum and mature milk adapted data from Kretchmer and Zimmermann (1997) [24]. 
Table 4 Comparison of the nutrient content of P-519 with dietary reference intake for infants and children.

\begin{tabular}{|c|c|c|c|c|}
\hline \multirow{3}{*}{ Nutrients } & \multicolumn{4}{|c|}{ Age (years) } \\
\hline & \multicolumn{2}{|l|}{ Infants } & \multicolumn{2}{|c|}{ Children } \\
\hline & $0-0.5$ & $0.5-1$ & $1-3$ & P-519 (per 100 g) \\
\hline Energy EER (Kcal/day) & 570 & 743 & 1046 & $95.96 \mathrm{mg}$ \\
\hline Protein RDA (g/kg/day) & 1.52 & 1.2 & 1.05 & $1.61 \mathrm{mg}$ \\
\hline Carbohydrate RDA (g/day) & 60 & 95 & 130 & $15.27 \mathrm{mg}$ \\
\hline Fat AI (g/day) & 31 & 30 & - & $3.16 \mathrm{mg}$ \\
\hline Potassium AI (mg/day) & 400 & 700 & 3000 & $4,790 \mathrm{mg}$ \\
\hline Magnesium RDA (mg/day) & 30 & 75 & 80 & $1,090 \mathrm{mg}$ \\
\hline Sodium AI (mg/day) & 120 & 370 & 1000 & 170 mg \\
\hline Calcium AI (mg/day) & 210 & 270 & 500 & $740 \mathrm{mg}$ \\
\hline Phosphorus RDA (mg/day) & 100 & 275 & 460 & $280 \mathrm{mg}$ \\
\hline Iron RDA (mg/day) & 0.27 & 11 & 7 & $7.98 \mathrm{mg}$ \\
\hline Zinc RDA (mg/day) & 2 & 3 & 3 & $17.4 \mathrm{mg}$ \\
\hline Copper RDA (mg/day) & 0.2 & 0.22 & 0.34 & $6.45 \mathrm{mg}$ \\
\hline Manganese AI (mg/day) & 0.003 & 0.6 & 1.2 & $8.9 \mathrm{mg}$ \\
\hline \multicolumn{5}{|c|}{$\begin{array}{l}\text { Source: Dietary Reference Intake series adapted from the National Academy Press (1997; 2001; 2005) [25, 26, 27]. EER- Estimat } \\
\text { Energy Requirements. }\end{array}$} \\
\hline Test & \multicolumn{2}{|c|}{ Count (cfu/mL) } & \multicolumn{2}{|c|}{ RUTF } \\
\hline Aerobic Plate Count & \multicolumn{2}{|c|}{$20(200)$} & \multicolumn{2}{|l|}{-} \\
\hline Coliform & \multicolumn{2}{|c|}{$3(30)$} & \multicolumn{2}{|c|}{ Negative in $1 \mathrm{~g}$} \\
\hline E. coli & \multicolumn{2}{|c|}{$0(<10)$} & \multicolumn{2}{|c|}{-} \\
\hline Yeast & \multicolumn{2}{|l|}{$8.5(85)$} & \multicolumn{2}{|c|}{ Maximum 10 in $1 \mathrm{~g}$} \\
\hline Moulds & \multicolumn{2}{|l|}{$0(<10)$} & \multicolumn{2}{|c|}{ Maximum 50 in $1 \mathrm{~g}$} \\
\hline
\end{tabular}

Source: Proposed Maximum Toxin level for RUTF adapted from WHO (2007) [3].

\subsection{Microbiological Analysis}

Severe malnourished children are already distressed by the condition. Proper hygiene practice therefore becomes imperative in the treatment and prevention of infections and to facilitate recovery. Food spoilage microorganisms and toxins can aggravate their condition or send them to their grave earlier if swallowed. This prompted the United Nation Organizations interested in nutrition and health to propose microbial load that should be found in RUTFs. In 2007, WHO recommended for all RUTF should contain; coliform (Negative in $1 \mathrm{~g}$ ), yeast (Maximum 10 in $1 \mathrm{~g}$ ) and mould (Maximum 50 in $1 \mathrm{~g}$ ) [3]. Respectively, comparing it with the microbial examination of P-519 (Table 5) suggest that P-519 was prepared in a very hygienic environment. No observable colonies E. coli and moulds were found
(Table 5).

\section{Conclusion}

The result of this study reveals that the nutrient content P-519 had the highest ratings among the three samples sensory evaluated and meets the proposed nutrient composition range for RUTF but the phosphorus and iron content did not. However, it has higher nutrient content than F-75 and prominent amount of sodium, zinc, magnesium and copper than F-100, F-135 and plumpy nut respectively which are already being used in severe malnutrition management. The microbiological examination revealed that P-519 had lower microbial content than that proposed for RUTF. But, further study is needed to test the potency of P-519 in rats and clinical trial in severely malnourished children (6-59 months). 


\section{References}

[1] UNICEF. 2012. "Levels \& Trends in Child Mortality." Estimates Developed by the UN Inter-agency Group for Child Mortality Estimation. New York: UNICEF.

[2] Access to Nutrition Index. 2013. Global index. 7-45. Available at: http://s3.amazonaws.com/ATN/atni_global_index_2013.p df. Accessed 23/08/2014.

[3] World Health Organization. 2007. "Community-Based Management of Severe Acute Malnutrition.” Geneva: World Health Organization, 3-8.

[4] UNICEF. June 2013. "Ready-to-Use Therapeutic Food for Children with Severe Acute Malnutrition.” Position paper No. 1.

[5] UNICEF. 2013. Improving Child Nutrition: The Achievable Imperative for Global Progress; Save the Children, Food for Thought.

[6] Black, R. E., Victora, C. G., Walker, S. P., Bhutta, Z. A., Christian, P., De Onis, M., and Uauy, R. 2013. "Maternal and Child Undernutrition and Overweight in Low-Income and Middle-Income Countries.” The Lancet 382 (9890): 427-51.

[7] Martins, V. J., Toledo Florêncio, T. M., Grillo, L. P., Do Carmo, P., Franco, M., Martins, P. A., Clemente, A. P. G., and Sawaya, A. L. 2011. "Long-Lasting Effects of Undernutrition.” International Journal of Environmental Research and Public Health 8 (6): 1817-46.

[8] Ghana Health Service. 2010. Interim National Guidelines for Community-Based Management for Severe Acute Malnutrition in Ghana.

[9] World Health Organization. 2003. "Guidelines for the In-patient Treatment for Severely Malnourished Children.” World Health Organization, 5-17.

[10] WHO/UNICEF. 2009. "WHO Child Growth Standards and the Identification of Severe Acute Malnutrition in Infants and Children: A Joint Statement by the World Health Organization and the United Nations Children's Fund.” World Health Organization (WHO), 4-15.

[11] UNICEF. 1998. "Monitoring and Evaluation Training Resource. Conceptual Framework: Causes of Child Malnutrition.” The State of the World's Children.

[12] Victora, C. G., Adair, L., Fall, C., Hallal, P. C., Martorell, R., Richter, L., and Sachdev, H. S. 2008. "Maternal and Child Undernutrition: Consequences for Adult Health and Human Capital.” The Lancet 371 (9609): 340-57.

[13] Latham, M. C., Jonsson U., Sterken E., and Kent, G.
2011. "RUTF Stuff: Can the Children Be Saved with Fortified Peanut Paste?” World Nutrition 2 (2): 62-85.

[14] UNICEF. 2010. Technical Requirement for RUTF Product.

[15] AOAC. 1990. Official Methods of Analysis of the Association of Official Analytical Chemists. 15th ed. Washington, DC: Association of Official Analytical Chemists, 341-8.

[16] AOAC. 2000. Officials Methods of Analysis of AOAC International. 17th ed. Method Nr 905.02. Gravimetric Method (Rose Gottlieb), USA, 208-15.

[17] AOAC International. 2000. Official Methods of Analysis of AOAC International. 17th ed. Gaithersburg, MD, USA: Association of Analytical Communities, 201-7.

[18] SPSS Inc. 2007. SPSS for Windows, Version 16.0. Chicago: SPSS Inc.

[19] Meilgaard, M. C., Carr, B. T., and Civille, G. V. 2006. Sensory Evaluation Techniques. CRC Press, 21-43.

[20] Svensson, Linn. 2012. Design and Performance of Small Scale Consumer Test. 354, 1-63. http://stud.epsilon.slu.se.

[21] Koppel, K., Chambers IV, E., Vázquez-Araújo, L., Timberg, L., Carbonell-Barrachina, Á. A., and Suwonsichon, S. 2014. "Cross-country Comparison of Pomegranate Juice Acceptance in Estonia, Spain, Thailand, and United States." Food Quality and Preference 31: 116-23.

[22] Action Contre La Faim International. 2011. “Guidelines for the Integrated Management of Severe Acute Malnutrition: In- and Out-patient Treatment.” http://www.actionagainsthunger.org/publication/guildines -integrated-management-severe-acute-malnutrition-and-o ut-patient-treatment. Accessed 20/09/2013.

[23] Golden, M. H. 2009. "Proposed Recommended Nutrient Densities for Moderately Malnourished Children.” Food \& Nutrition Bulletin 30 (3): S267.

[24] Kretchmer, N., and Zimmermann, M. 1997. Developmental Nutrition. Boston: Allyn and Bacon, 2-10.

[25] Committee on Dietary Reference Intakes. 1997. Dietary Reference Intakes for Calcium, Phosphorus and Magnesium. Washington D.C.: National Academy Press.

[26] Committee on Dietary Reference Intakes. 2001. Dietary Reference Intakes for Copper, Iron, Manganese and Zinc. Washington, D.C.: National Academy Press.

[27] Committee on Dietary Reference Intakes. 2005. Dietary Reference Intakes for Potassium, Sodium, Carbohydrate, Fat, Protein and Energy. Washington, D.C.: National Academy Press. 